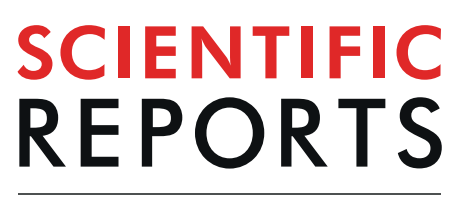

natureresearch

\title{
OPEN Field cress genome mapping: Integrating linkage and comparative maps with cytogenetic analysis for rDNA carrying chromosomes
}

\author{
Zeratsion Abera Desta ${ }^{1 *}$, Bozena Kolano $\mathbb{D}^{2}$, Zeeshan Shamim ${ }^{3,4}$, Susan J. Armstrong $\mathbb{1}^{4}$, \\ Monika Rewers $\mathbb{D}^{5}$, Elwira Sliwinska ${ }^{5}$, Sandeep Kumar Kushwaha ${ }^{1}$, Isobel A. P. Parkin ${ }^{6}$, \\ Rodomiro Ortiz ${ }^{1} \&$ Dirk-Jan de Koning $\mathbb{1}^{7}$
}

Field cress (Lepidium campestre L.), despite its potential as a sustainable alternative oilseed plant, has been underutilized, and no prior attempts to characterize the genome at the genetic or molecular cytogenetic level have been conducted. Genetic maps are the foundation for anchoring and orienting annotated genome assemblies and positional cloning of candidate genes. Our principal goal was to construct a genetic map using integrated approaches of genetic, comparative and cytogenetic map analyses. In total, $503 \mathrm{~F}_{2}$ interspecific hybrid individuals were genotyped using 7,624 single nucleotide polymorphism markers. Comparative analysis demonstrated that $\sim 57 \%$ of the sequenced loci in $L$. campestre were congruent with Arabidopsis thaliana (L.) genome and suggested a novel karyotype, which predates the ancestral crucifer karyotype. Aceto-orcein chromosome staining and fluorescence in situ hybridization (FISH) analyses confirmed that L. campestre, L. heterophyllum Benth. and their hybrids had a chromosome number of $2 n=2 x=16$. Flow cytometric analysis revealed that both species possess 2 roughly 0.4 picogram DNA. Integrating linkage and comparative maps with cytogenetic map analyses assigned two linkage groups to their particular chromosomes. Future work could incorporate FISH utilizing $A$. thaliana mapped BAC clones to allow the chromosomes of field cress to be identified reliably.

The genus Lepidium consisting of 231 species is one of the largest of 338 genera in the Brassicaceae (Cruciferae or Mustard) family ${ }^{1}$. Field cress (Lepidium campestre L.) is a self-pollinated, diploid $(2 n=2 x=16)$ biennial plant originating in Europe ${ }^{2}$ and subsequently appearing as an introduced weed in North America. Smith's pepperwort (Lepidium heterophyllum Benth.) is a diploid $(2 n=2 x=16)$ perennial plant, and has relatively lower winter hardiness than L. campestre ${ }^{3}$.

L. campestre has potential as an oilseed $\mathrm{crop}^{3,4}$, and could provide an alternative intercrop species with members of the Poaceae family (e.g. barley) ${ }^{5}$. Following the harvest of the main crop, field cress can be engaged as a catch crop during the off-season to prevent soil nutrient leaching ${ }^{6}$, in turn this alleviates underground environmental pollution. Furthermore, field cress could reduce fertilizer dependency (e.g. nitrogen fertilizer), which maintains the soil quality, thereby mitigating the current and future challenges of climate change. These

${ }^{1}$ Department of Plant Breeding, Swedish University of Agricultural Sciences, Sundesvagen 10, Box 101, SE23053, Alnarp, Sweden. ${ }^{2}$ Department of Plant Anatomy and Cytology, University of Silesia, Jagiellonska 28, 40032, Katowice, Poland. ${ }^{3}$ Mirpur University of Science and Technology (MUST), Mirpur AJK, Pakistan. ${ }^{4}$ School of Biosciences, University of Birmingham, Birmingham, B 15 2TT, United Kingdom. ${ }^{5}$ Laboratory of Molecular Biology and Cytometry, Department of Agricultural Biotechnology, UTP University of Science and Technology, Kaliskiego Ave. 7, 85-789, Bydgoszcz, Poland. ${ }^{6}$ Agriculture and Agri-Food Canada, 107 Science Place, Saskatoon, SK, S7N0X2, Canada. ${ }^{7}$ Department of Animal Breeding and Genetics, Swedish University of Agricultural Sciences, Box 7023, SE 75007, Uppsala, Sweden. *email: zeratsion.abera@slu.se 
foundational features of the species suggest the possibilities that could arise from domestication of field cress which has yet been underutilized and has been the focus of limited research.

There have been substantial advances in the ability to generate draft genome assemblies for many crop species, as well as in dense genotyping platforms to underpin whole-genome prediction ${ }^{7}$. However, the importance of genetic mapping and molecular cytogenetics remain undiminished, both in supporting these technologies, and in addressing fundamental biological questions related to genome and karyotype evolution. To date, neither a linkage map nor molecular cytogenetic analysis has ever been developed for L. campestre. Indeed, cytological investigations of Lepidium species have been mainly restricted to chromosome counts ${ }^{1,8}$. Lepidium like many of the genera in the Brassicaceae family (e.g., Arabidopsis and Cardamine) ${ }^{9,10}$ has small and poorly differentiated chromosomes that complicate karyotyping efforts. Hence, additional landmarks are required to identify chromosome pairs. The markers most often used are the $5 \mathrm{~S}$ and $45 \mathrm{~S}$ rDNA sequences due to their abundance in the genome and their relatively conserved nature ${ }^{11,12}$.

Meiotic recombination, the reshuffling of genes derived from ancestral gametes during meiosis, allows segregation of parental alleles to be detected in zygote progeny, and hence the inference of order and distance of genes to generate a genetic linkage map that represents a model of the physical chromosomes. Thus, the more meiotic recombination events are captured, the better the resolution of the linkage map ${ }^{13}$. Successful plant improvement strategies predominantly rely on the distribution of crossovers between homologous chromosomes ${ }^{14,15}$. Linkage maps are further employed to detect quantitative trait loci (QTL), to anchor and orient annotated genome assemblies, and to locate candidate genes in fine mapping.

The availability of reference sequences in model plants - such as thale cress (Arabidopsis thaliana L.) - sheds contemporary light on the underlying mechanisms of genome architecture evolution. Anchoring linkage maps to the A. thaliana genome uncovered highly conserved genomic regions in Brassica species ${ }^{16,17}$, indicating that a linkage map could serve as a supplementary toolkit in comparative genomics to translate genomic information between related taxa. Here we analysed with 7,624 single nucleotide polymorphism (SNP) markers the genomes of $501 \mathrm{~F}_{2}$ interspecific hybrid individuals resulting in a map with eight linkage groups (LGs). Integrating the SNP loci of field cress into the Arabidopsis genome revealed a high proportion ( $\sim 57 \%)$ of sequenced loci were found in regions of conserved synteny between species. We further intertwined the linkage and comparative maps with cytogenetic map, and the cooperative actions of these techniques posited the allocation of LGs into their chromosomes.

Towards the plant domestication of a potentially valuable oilseed plant, we describe herein the aims of (i) developing a linkage map and assigning LGs to their chromosomes in L. campestre using integrating techniques of genetic, comparative, and cytogenetic map analyses; (ii) estimating the genome size of L. campestre and $L$. heterophyllum; (iii) conducting molecular cytogenetic analysis in L. campestre, L. heterophyllum, and their hybrid individuals; and (iv) identifying the ancestral genomic block (GB) structure for L. campestre.

\section{Results and Discussion}

Genome size estimation. The 2C DNA content of the $54 \mathrm{~L}$. campestre accessions ranged from 0.394 $\mathrm{pg} / 2 \mathrm{C}$ to $0.439 \mathrm{pg} / 2 \mathrm{C}$, which corresponded to 385 megabase pairs (Mb)/2C and $429 \mathrm{Mb} / 2 \mathrm{C}$, respectively (Supplementary Table 1, Supplementary Fig. 1). The mean genome sizes of L. campestre and L. heterophyllum were $0.416 \mathrm{pg} / 2 \mathrm{C}(407 \mathrm{Mb} / 2 \mathrm{C})$ and $0.411 \mathrm{pg} / 2 \mathrm{C}(402 \mathrm{Mb} / 2 \mathrm{C})$, respectively. For L. heterophyllum, this is the first report of genome size estimation. Despite differences in their growth habits, there was no significant difference in mean DNA content between studied species ([ANOVA] $F=1.918$, degree of freedom $[\mathrm{df}]=1, P=0.172$ ). According to the proposed categorization ${ }^{18}, L$. campestre and L. heterophyllum possess very small genomes $(<2.8 \mathrm{pg} / 2 \mathrm{C})$. A previous report for L. campestre genome size $(0.7 \mathrm{pg} / 2 \mathrm{C})^{19}$ and other prior estimates for higher ploidy Lepidium species - ranging between 0.66 and $2.08 \mathrm{pg} / 2 \mathrm{C}^{20}$ - were higher than the estimate established here.

Chromosome counting and fluorescence in situ hybridization (FISH) analysis. In addition to chromosome counting in L. campestre and L. heterophyllum with a standard acetoorcein chromosome staining method, we performed FISH analysis, and both species including their hybrids had $2 n=2 x=16$ chromosomes (Fig. 1a,d-f), in accordance with the published indexes (http://ccdb.tau.ac.il/Angiosperms/Brassicaceae/ Lepidium/; http://www.tropicos.org/Project/IPCN). Their karyotypes contained mostly metacentric and sub-metacentric chromosomes, which showed a gradual decrease in their length, except one pair of homologues, which was remarkably longer than the others (Figs 1a,d, 2).

This is the first report of rDNA localization in chromosomes of L. campestre, L. heterophyllum as well as their hybrids. rDNA sequences can be localized by FISH and can provide cytogenetic markers that are useful for the identification of individual chromosomes ${ }^{11,21}$. Similar to previously described L. africanum $(2 n=2 x=16)$ and many other angiosperms ${ }^{22,23}$, in all analysed Lepidium samples (Fig. 1a,d-f), we found that $5 \mathrm{~S}$ and $45 \mathrm{~S}$ rDNA loci were localized on different chromosomes. Two hybridization signals for $45 \mathrm{~S}$ rDNA were observed on mitotic metaphase chromosomes of $L$. campestre and L. heterophyllum (Fig. 1a,d,e). In many angiosperms, $45 \mathrm{~S} \mathrm{rDNA}$ loci were most often found in sub-terminal regions of the short arm of the chromosomes ${ }^{23}$. Such localization of $45 \mathrm{~S}$ rDNA loci was also reported in other Lepidium species ${ }^{22}$; however, L. campestre and L. heterophyllum revealed $45 \mathrm{~S}$ rDNA loci, both on the short and long arm of the same chromosome, in a more proximal location. To delineate the chromosomal localization of the rDNA, mitotic pro-metaphase chromosomes and meiotic (pachytene and metaphase) chromosomes were probed using FISH. The hybridization signals of 45S rDNA seem to cover most of the short arm, but also span to the proximal part of the long arm in the longest chromosome of L. campestre as seen at the pachytene stage, when the chromosomes are paired up and most extended (Figs 1a-c and 2).

The proximal localization of $5 \mathrm{~S}$ rDNA loci was frequently reported in karyotypes of angiosperms with small chromosomes $^{23}$. As in previous research of other Lepidium species ${ }^{22}$, our analysis revealed the 5 S rDNA locus in the interstitial position of the short arm of the sub-metacentric chromosomes (Figs 1a,b and 2). In all analysed 

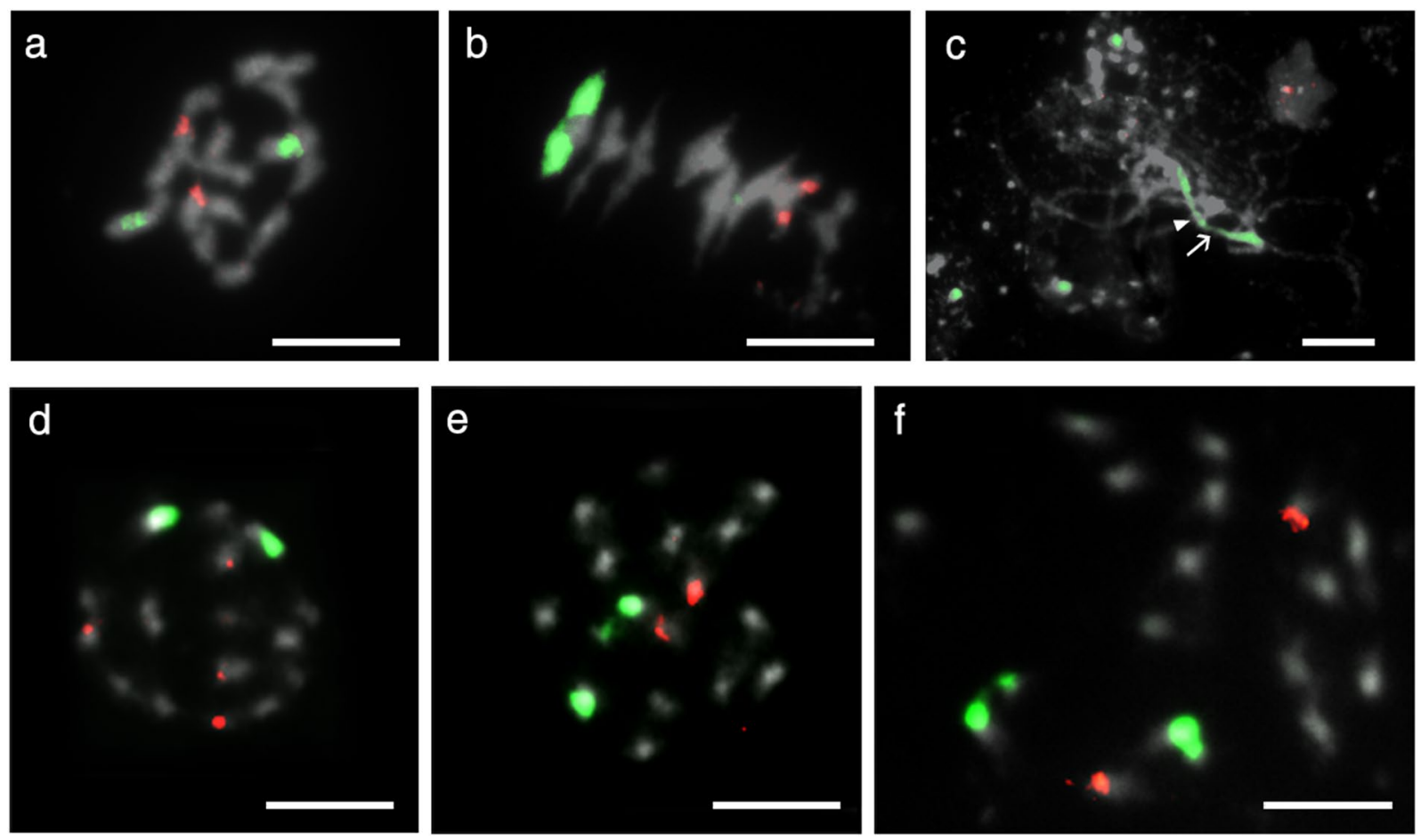

Figure 1. Chromosomal localization of $45 \mathrm{~S}$ rDNA loci (green fluorescence) and $5 \mathrm{~S}$ rDNA loci (red fluorescence) in Lepidium chromosomes. (a) Somatic metaphase. (b,c) meiotic chromosomes of L. campestre accession coded C54_1 (Supplementary Table 2). The arrowhead and arrow in (c) identify the centromere and secondary construction, respectively of a complete chromosome 1 bivalent in an incomplete pachytene cell. (d,e) Metaphase plate of L. heterophyllum accessions coded as C66_4 and BP1_1, respectively (Supplementary Table 2). (f) Somatic metaphase of a hybrid from L. campestre and $\bar{L}$. heterophyllum coded as Hy56_1. Bars represent $5 \mu \mathrm{m}$.

field cress accessions, only one 5S rDNA locus was detected, whereas L. heterophyllum showed intraspecific polymorphism in 5S rDNA locus number, depending on the accession type, one pair (sample code BP1_1) or two pairs (sample code C66_4) of the loci were recorded (Fig. 1a,d,e; Supplementary Table 2). Such a phenomenon was described in many plant genera ${ }^{11,21}$. In addition to the epigenetic nature of the specific locus (5S rDNA) ${ }^{24}$, various mechanisms have been postulated to account for this state such as a transposon-mediated transposition event or chromosome rearrangements caused by homologous or a non-homologous unequal crossing-over and gene conversion $^{25-27}$. Taken together, these results may reflect the high levels of micro-evolutionary or epigenetic changes within these taxa. Furthermore, in a hybrid individual (coded as Hy56_1) (Supplementary Table 2) one pair of $45 \mathrm{~S}$ rDNA and one pair of $5 \mathrm{~S}$ rDNA loci were detected (Fig. 1f). Interestingly, the karyotypes of all analysed accessions of Lepidium - in both species as well as their hybrid - were similar to each other.

Genetic linkage map construction. In total, $503 \mathrm{~F}_{2}$ interspecific hybrid individuals derived from two $\mathrm{F}_{1}$ parents (see methods section) were genotyped using 7,624 SNP markers. Segregating SNP loci common to both sub-populations were analysed, and these were combined with the uniquely segregating SNPs from each sub-population. In a combined linkage analysis, 2,016 of the 7,624 SNP markers were scored in 487 individuals. After quality control (see methods section for details), 1,517 SNPs were employed in $482 \mathrm{~F}_{2}$ individuals to generate a genetic linkage map for L. campestre (Fig. 3a; Supplementary Fig. 3). In the final mapping, 1,401 segregating SNP loci were common to both sub-populations, whereas 116 SNP loci were unique to one or other sub-population (Table 1).

The distribution of SNP loci varied in terms of number, density, and distance across LGs. The number of SNPs per LG ranged from the largest LG1 (434) to the smallest LG8 (60) (Table 1). The eight LGs (Fig. 1a, Supplementary Fig. 2) spanned 566.07 centimorgans (cM), with individual LG lengths of $24 \mathrm{cM}$ (LG8) to $115.90 \mathrm{cM}$ (LG7), and a mean density of three SNPs per cM (one SNP per $0.35 \mathrm{cM}$ ). The average distance between adjacent SNP loci ranged from $0.18 \mathrm{cM}$ in LG1 to $0.69 \mathrm{cM}$ in LG2, with a mean of $0.42 \mathrm{cM}$ (Table 1). At least one chiasma per LG were captured except for LG8, as this was smaller than $50 \mathrm{cM}$. For this LG, we are presumably missing some markers because for all of the metaphase I bivalents we have always seen at least one crossover.

Segregation distortion. A total of $483(\sim 32 \%)$ mapped SNP loci deviated significantly (linkage test: $P<0.05)$ from an expected Mendelian segregation ratio of 1:2:1 (Table 1). The highest number of distorted loci were on LG6 (181), followed by LG7 (102), whereas no distorted loci were found on LG1, LG5, and LG8. Skewed segregation of loci is not uncommon in interspecific hybrids, as already well exemplified in crops such as 


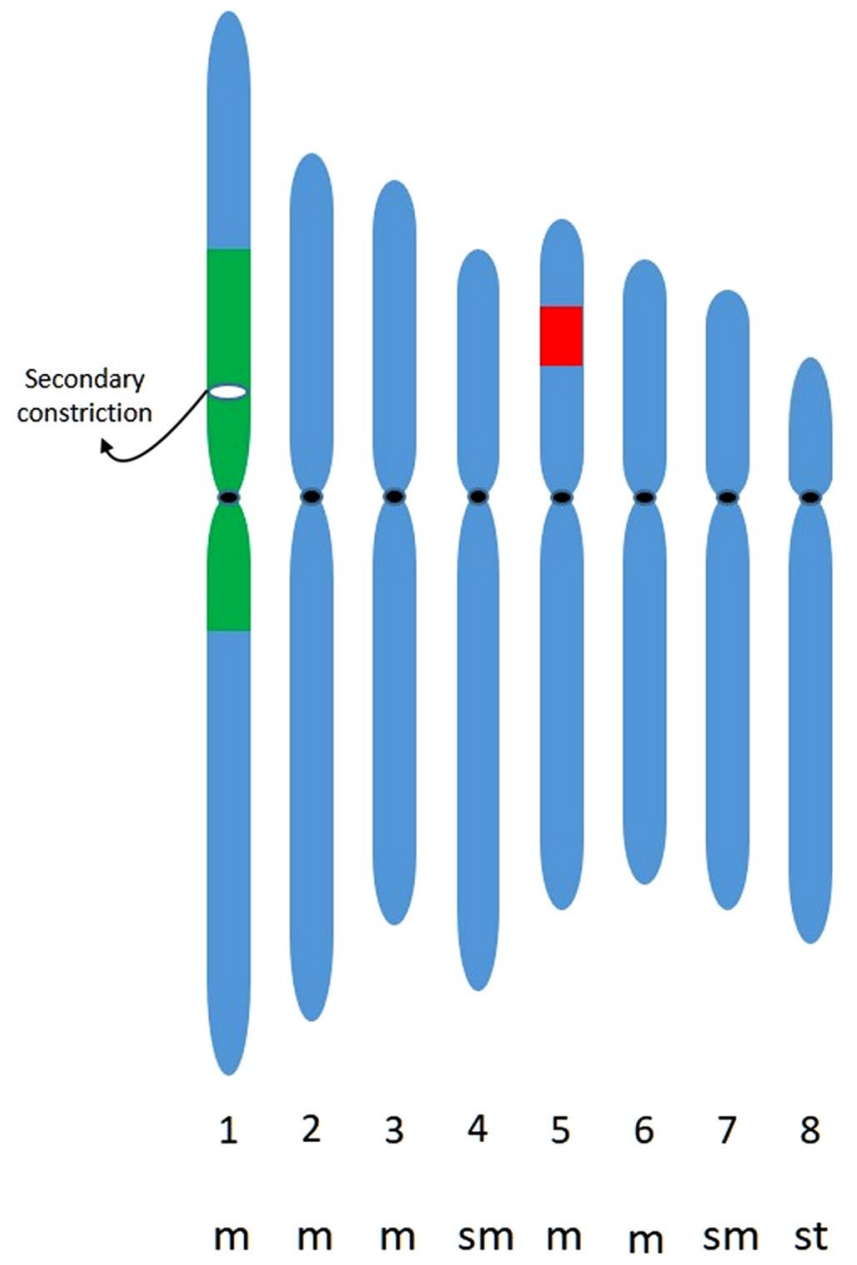

Figure 2. Haploid idiogram representing Lepidium campestre. The location of the $45 \mathrm{~S}$ rDNA (green) and the $5 \mathrm{~S}$ rDNA (red) are indicated on chromosome 1 and 5, respectively. A black line represents the centromeres, whereas a white line symbolizes the secondary constriction. Abbreviations: m: metacentric chromosome; sm: sub-metacentric chromosome; st: sub-telocentric chromosome.

$\operatorname{cotton}^{28,29}$ and rice ${ }^{30}$. Notably, the genetic causes of segregation abnormalities remain largely enigmatic. A number of hypotheses have been proposed to explain such events, for example the degree of divergence of the parental lines $^{31}$, or selection during gamete ${ }^{32}$ or zygote formation ${ }^{33}$. Regardless of segregation distortion, the relative order of loci in linkage maps remains unaffected ${ }^{34}$. Indeed, the inclusion of segregation distortions in highly distant crosses has biological relevance to circumvent further differentiation of $\mathrm{LGs}^{35}$. Nevertheless, strict checking of marker data is imperative to control for the spurious association of loci while fitting loci with deviant segregation patterns.

Evaluation of genetic linkage map. To evaluate the position and distribution of loci within and between LGs, we used various visualization plots. The presence of singletons not only creates erroneous artefacts, but can also merges two unrelated LGs together ${ }^{35}$. When marker loci are separated with a maximum gap of $\sim 40 \mathrm{cM}$ in the same homologous chromosomes, these markers could actually be unlinked and form different chromosomes $^{36}$. In light of this, we refined the clustering of loci on the basis of patterns apparent in $2 \mathrm{D}$ non-parametric multi-dimensional scaling ( $n p$ MDS) ordination plots (Fig. 4a), and hierarchical clustering dendrograms (Fig. 4b) to potentially resolve spurious linkages. In agreement with linkage mapping, the $n p M D S$ identified the same pattern and distribution of loci within and between LGs, while clustering dendrograms highlighted the distinction of loci between LGs.

To gain additional insight, we computed significant strong cross link (SCL) values (LOD threshold value $>3$ to verify LGs in genetic mapping ${ }^{35,37}$. Based on these values, Fig. 3 b reveals the interplay between the sub-groups of the potentially sub-divided LGs (LG5 and LG7) with persistent routes found between them, reflecting that the subdivided LGs were the fusion of their original LGs designation. To scrutinize this further, we explored the relative map position of loci using higher and then lower stringency LOD threshold values ${ }^{35}$, although this approach is less reliable. In this context, we noted similar loci orders (Supplementary Table 3) between the highly stringent (LOD threshold values $\geq 9$ and $\geq 11$ for LG5 and LG7, respectively) and the less stringent LOD threshold values 


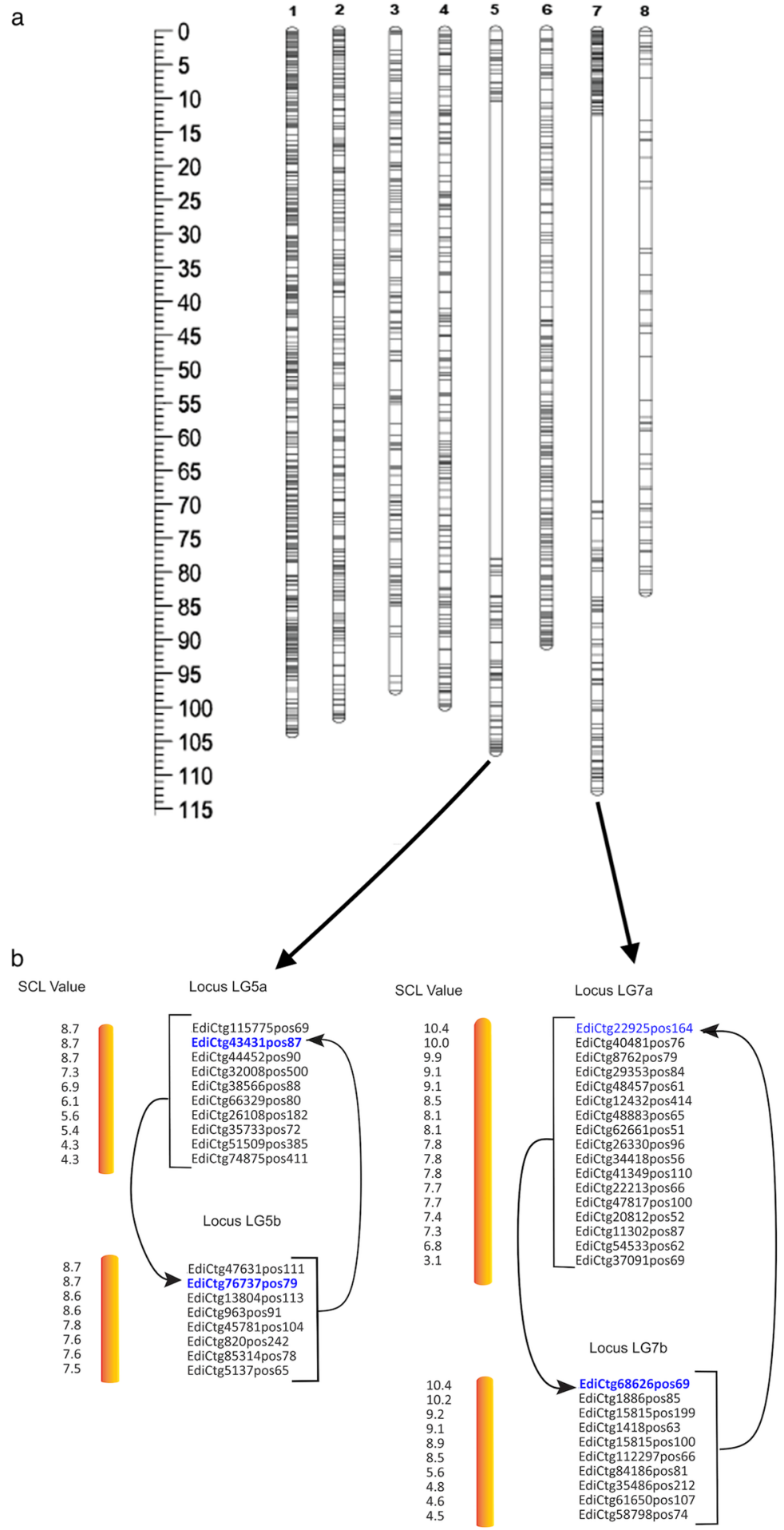

Figure 3. Genetic linkage map constructed for field cress. (a) The expected eight linkage groups (LGs) constructed using 1,517 SNP markers. The left side ruler is highlighted to indicate the distance intervals between adjacent loci. (b) The strong cross-link (SCL) values used to indicate the loop interplaying routes of loci between the potentially fragmented LG5 and LG7 related to their sub-LGs (Sub-LG5a, sub-LG5b, and subLG7a, sub-LG7b, respectively). The subdivision of LGs existed under logarithms of the odds (LOD) threshold values $\geq 9$ and $\geq 11$ for LG5 and LG7, respectively. The locus highlighted in blue is an entry to a group of loci from the other sub-divided LG.

(LOD threshold values $<9$ for LG5, $<11$ for LG7), lending additional support that the resulting fragments within these LGs (Fig. 1b) should not be the split into independent LGs. Moreover, the above evidence was corroborated with molecular cytogenetic studies (see the results of the cytogenetic section). Given the similarity karyotype 


\begin{tabular}{|c|c|c|c|c|c|c|c|c|}
\hline \multirow[b]{2}{*}{ LG } & \multicolumn{3}{|c|}{ Number of marker locus } & \multirow{2}{*}{$\begin{array}{l}\text { Map length } \\
(c M)\end{array}$} & \multirow{2}{*}{$\begin{array}{l}\text { Average } \\
\text { distance }(c M)\end{array}$} & \multirow{2}{*}{$\begin{array}{l}\text { Max. } \operatorname{gap}^{c} \\
(c M)\end{array}$} & \multirow[b]{2}{*}{$\mathrm{SNP} / \mathrm{cM}$} & \multirow[b]{2}{*}{ Sg. $D^{d}$} \\
\hline & Com. $^{\mathrm{a}}$ & Uniq. $^{b}$ & Total & & & & & \\
\hline 1 & 429 & 5 & 434 & 76.35 & 0.18 & 1.36 & 5.7 & - \\
\hline 2 & 217 & - & 217 & 75.03 & 0.69 & 2.23 & 3.0 & 45 \\
\hline 3 & 136 & 16 & 152 & 60.06 & 0.40 & 3.60 & 2.5 & 85 \\
\hline 4 & 141 & 42 & 183 & 73.99 & 0.40 & 1.80 & 2.5 & 70 \\
\hline 5 & 85 & 0 & 85 & 93.36 & 0.39 & 59.18 & 1.0 & - \\
\hline 6 & 161 & 52 & 213 & 47.38 & 0.22 & 1.25 & 4.5 & 181 \\
\hline 7 & 172 & 1 & 173 & 115.90 & 0.67 & 58.84 & 1.5 & 102 \\
\hline 8 & 60 & - & 60 & 24.00 & \begin{tabular}{|l|}
0.40 \\
\end{tabular} & 2.56 & 2.5 & - \\
\hline Total & 1,401 & 116 & 1,517 & 566.07 & - & - & - & 483 \\
\hline Mean & 175.13 & 14.50 & 189.63 & 70.76 & 0.42 & - & - & - \\
\hline
\end{tabular}

Table 1. Summarized distribution of marker loci, map length, and segregation distortion in each linkage group (LG) of field cress a SNP locus common to both sub-populations, bSNP locus unique to one or other subpopulation, ${ }^{\mathrm{c}}$ maximum gap, ${ }^{\mathrm{d}}$ segregation distortion.

analyses across all samples, we reasoned that the resulting gaps within both LG5 and LG7 possibly stemmed from ancestral rearrangements of chromosomes in both Lepidium species (L. campestre and L. heterophyllum), and have existed after their evolutionary divergence of the two species.

Comparative map analysis. The local alignment search tool nucleotide (BLASTN) search using L. campestre SNP sequences showed $\sim 83 \%(1,254)$ of the 1,517 SNP loci shared sequence similarity with the A. thaliana genome (Table 2; Supplementary Table 4). However, the presence of sequence similarity or BLAST hits between species does not necessarily reflect their syntenic relationships ${ }^{38}$. In our comparative map analysis, $\sim 69 \%(866)$ of the 1,254 similar sequence loci (or $\sim 57 \%$ of the 1,517 polymorphic sequence loci) were congruent with the Arabidopsis genome (Fig. 5; Table 2). The relative reduction in homologous loci (from $\sim 69 \%$ to $~ 57 \%$ ) probably due to the occurrence of translocation, fusion or fission of genes after the evolutionary divergence of these two species $^{39}$. However, the 866 homologous loci identified highly conserved regions of the genomes after the evolutionary divergence of the two species. The number of conserved syntenic loci per LG varied between 36 (LG5) and 280 (LG1) (Table 2).

Comparative mapping is not only used to elucidate QTL bearing regions, but also to engage in positional cloning of genes ${ }^{40,41}$. The characterization of flowering time in the Arabidopsis genome is one of the notable examples in translating genomics between related species. The Flowering Locus C (FLC) gene has been found to underlie a major domestication QTL, which inhibits flowering in plants ${ }^{42,43}$. The orthologous analyses of FLC genes unveiled common regions between Brassica species and Arabidopsis genome ${ }^{44,45}$. Since ancestrally conserved regions typically harbour functional genes, mapping genomic regions and exploiting known synteny relationships, could be a keystone to accelerate the future domestication and improvement of field cress.

Next, we quantified 60 insertion - deletions (indels) that ranged from two on LG5 to 18 on LG1 (Table 2, Supplementary Table 4). We speculate that the presence of only a few indels might be either because of losses in diversity (e.g., small effective population size $)^{46}$ or because of the short evolutionary history of divergence accompanied by slow evolution rates of indel polymorphisms ${ }^{47}$. To this end, however, we note further efforts are needed for gaining an in-depth understanding of the underlying genomic plasticity that result in indel variation.

The comparative study between field cress and Arabidopsis yielded 46 nuclear duplications in seven of the eight LGs (Table 2, Supplementary Table 5). Lui and Adam ${ }^{48}$ found the expression of SHORT SUSPENSOR (SSP) gene was retained following duplication, suggesting neofunctionalization (new functions) in genes controlling brassinosteroid signal transduction of Brassica species. The Conserved Telomere Maintenance Component 1 (CTC1) was one of the paradigmatic duplications in L. campestre found in LG1 (Supplementary Table 4); however, further functional validation of the CTC1 gene is crucial. In higher plants, the interaction of CTC1 with SRN1 led to a new function that integrates and maintains the telomere regions of the chromosome ${ }^{49}$. Collectively, these findings indicate that exploring the conserved syntenywith $A$. thaliana can provide biological insights to unlock genes that underlie traits of interest in field cress.

Defining the ancestral genomic block structure for field cress. The study of genome evolution within the Brassicaceae has been facilitated by the definition of 24 ancestral genomic blocks (GBs), A- X, that are identified based on conserved gene content and order across all species of the family studied to date ${ }^{50,51}$. Utilizing the conserved synteny mapped between $A$. thaliana and $L$. campestre genomes, it was possible to identify 21 of the 24 GBs (Fig. 6, Supplementary Table 6), three of the blocks (G, S and T) were not found, which can be explained by their consistent peri-centromeric location that often leads to a paucity of useful polymorphic markers. $L$. campestre in most recent studies has been placed at the base of clade A (or lineage I) of the Brassicaceae ${ }^{52}$ and would be expected to have evolved from the previously characterized ancestral crucifer karyotype (ACK). Indeed, six of the L. campestre linkage groups share a common GB structure with six of the ACK chromosomes (Fig. 6). However, LG1 and LG2 of L. campestre are rearranged from two ACK chromosomes (AK6 and AK8) and most interestingly show ancestral GB associations (R-W and Q-X; Fig. 6) that have not been documented in 18 previously studied clade A species and were thought to be indicative of species from clade $\mathrm{B}^{51}$. The karyotype of field 


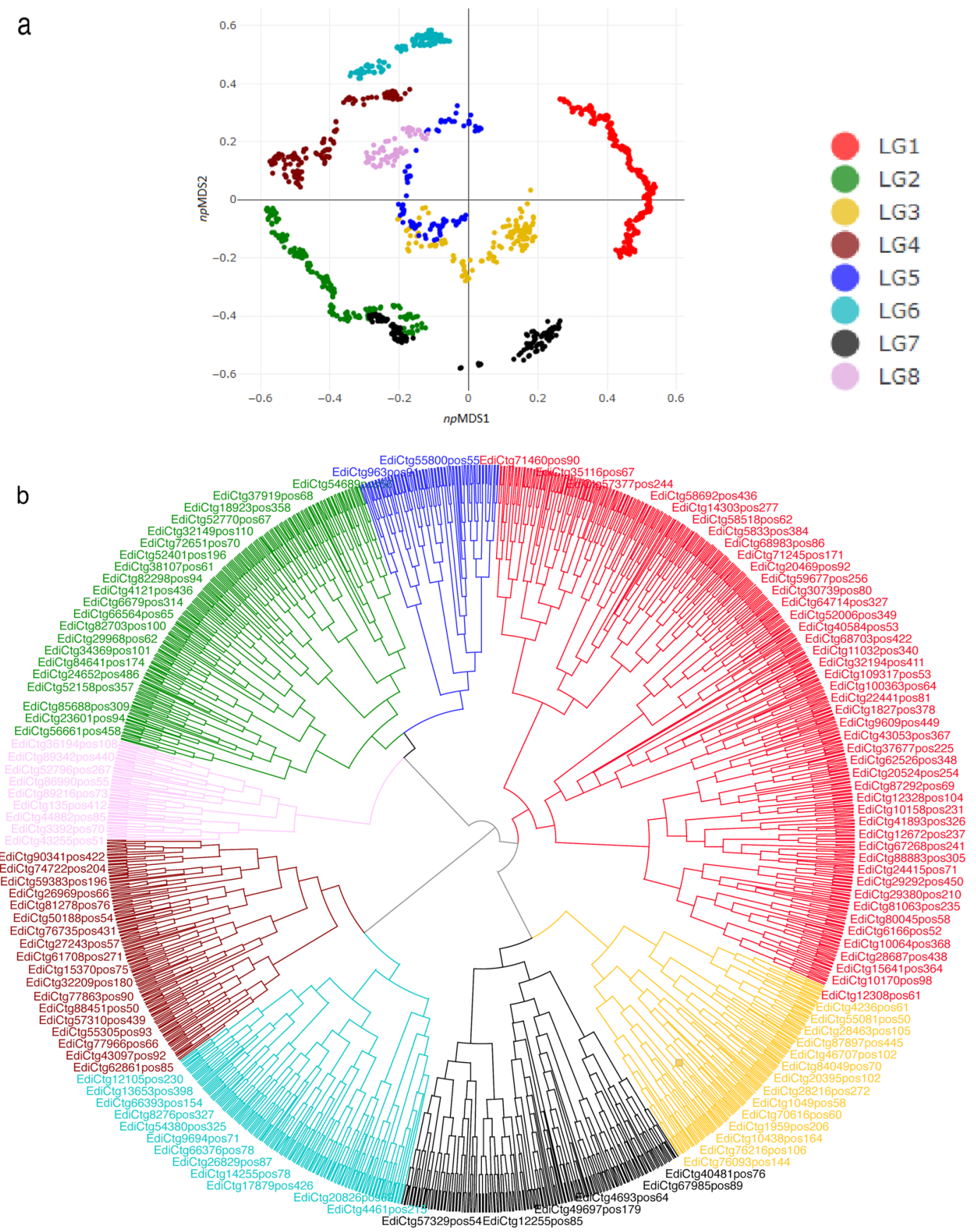

Figure 4. Visualization of loci distribution between and within linkage groups (LGs) of Lepidium campestre. (a) The 2D $n p$ MDS plot used to distinguish the structure and distribution of loci between and within LGs. (b) Hierarchal cladogram clustering employed to visualize loci positions between and within LGs.

cress effectively links clade A and B and the basal position of the species within clade A suggests that this novel karyotype pre-dates the ACK.

Assigning LGs to their chromosomes. A key challenge in genetic mapping is the reliable alignment of LGs to their chromosomes. This becomes even more problematic when genotyping is done in the absence of a reference genome, which is the case for L. campestre in this study. Thus, additional techniques such as cytogenetic and physical map analyses could reliably orient LGs to their chromosomes. Given the cytogenetic analysis, the present findings are helpful for an initial overview to assign LGs to their chromosomes. To estimate the LG map based on diakinesis or metaphase I cells, we inferred the number of crossovers and found a mean crossover number of 13 , equivalent to $650 \mathrm{cM}$ (13 multiplied by $50 \mathrm{cM})$. 


\begin{tabular}{|c|c|c|c|c|c|c|c|c|c|c|c|}
\hline \multirow[b]{2}{*}{$\mathbf{L G}^{\mathbf{a}}$} & \multirow{2}{*}{$\begin{array}{l}\text { Marker } \\
\text { locus }\end{array}$} & \multirow{2}{*}{$\begin{array}{l}\text { Blast } \\
\text { hit }\end{array}$} & \multirow[b]{2}{*}{ Ins $^{b}$} & \multirow[b]{2}{*}{ Del $^{\mathrm{c}}$} & \multirow[b]{2}{*}{ Dup $^{d}$} & \multirow{2}{*}{$\begin{array}{l}\text { Homol. } \\
\text { loci }^{\mathrm{e}}\end{array}$} & \multicolumn{5}{|c|}{$\begin{array}{l}\text { Loci homologous to A. thaliana } \\
\text { chromosomes }\end{array}$} \\
\hline & & & & & & & Chri $^{\mathrm{f}}$ & Chr2 & Chr3 & Chr4 & Chr5 \\
\hline LG1 & 434 & 356 & 6 & 13 & 22 & 280 & - & 2 & - & 109 & 169 \\
\hline LG2 & 217 & 179 & 5 & 5 & 4 & \begin{tabular}{|l|}
107 \\
\end{tabular} & - & - & - & - & 107 \\
\hline LG3 & 152 & 135 & 2 & 6 & 6 & 96 & 96 & - & - & - & - \\
\hline LG4 & 183 & 144 & 4 & 1 & 2 & 74 & - & 14 & 60 & - & - \\
\hline LG5 & 85 & 70 & 1 & 1 & - & 36 & - & 9 & 27 & - & - \\
\hline LG6 & 213 & 168 & 4 & 4 & 4 & 127 & 127 & - & - & - & - \\
\hline LG7 & \begin{tabular}{|l|}
173 \\
\end{tabular} & 146 & 1 & 3 & 6 & $\begin{array}{l}104 \\
\end{array}$ & - & 104 & - & - & - \\
\hline LG8 & 60 & 56 & 3 & 1 & 2 & 42 & - & - & - & 42 & - \\
\hline Total & 1,517 & 1,254 & 26 & 34 & 46 & 866 & 233 & 129 & 87 & 151 & 276 \\
\hline
\end{tabular}

Table 2. The 1,517 sequence loci in eight linkage groups (LGs) of field cress related to indels, duplications, collinearity, and homology with Arabidopsis genome alinkage group, binsertions, 'deletions, dduplications, ehomologous loci, ${ }^{\mathrm{f}}$ A . thaliana chromosome.

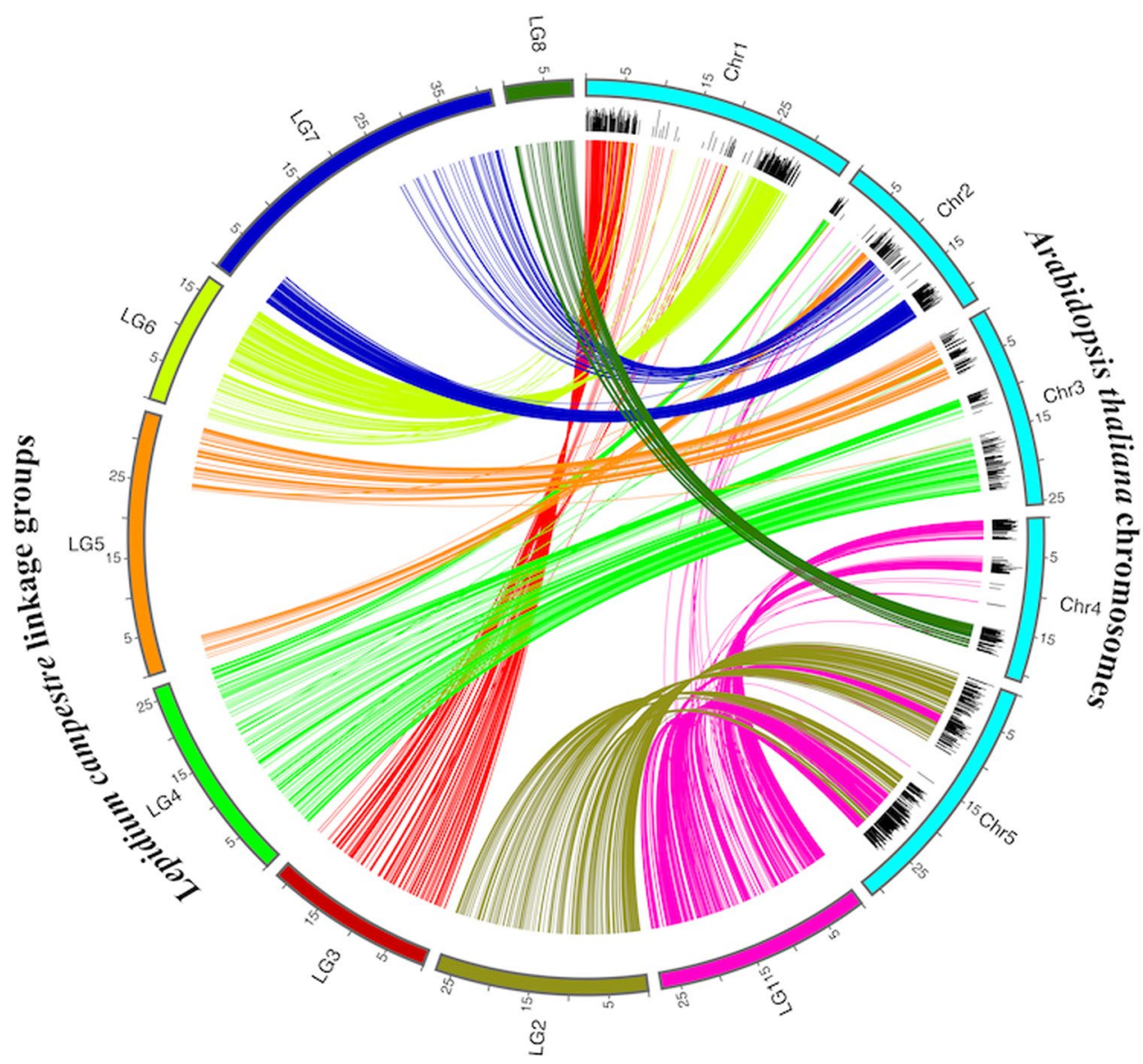

Figure 5. Graphical representation of sequence similarity that reveals the conserved syntenic regions between linkage groups (LGs) of field cress and chromosomes of Arabidopsis thaliana. The black bars show the level of similarity of SNP loci against the Arabidopsis genome.

The relatively small reduction (13\%) in size between genetic map (566.07 cM) (Table 1$)$ and cytogenetic map $(650 \mathrm{cM})$ (Fig. 5) is not unexpected, as linkage is highly sensitive to various factors such as accuracy of genotype scoring, representation of recombinants in the population, density of markers, and other constraints of mapping estimations $^{53}$. This is exemplified by LG8 at $24 \mathrm{cM}$, which could result from a shortage of polymorphic markers. A minimum of one crossover would be expected because of the requirement that at least one obligate crossover (a size of $50 \mathrm{cM}$ ) was needed for normal segregation and perfect fertility ${ }^{54}$. Thus, the LG map is likely to be expanded as more markers become available.

Extrapolating chromosome 1 from the defined karyotype (Fig. 2) suggests it is highly likely related to LG7 for the following reasons. First, chromosome 2 in A. thaliana has a large site for $45 \mathrm{~S} \mathrm{rDNA}$, and it is evident 


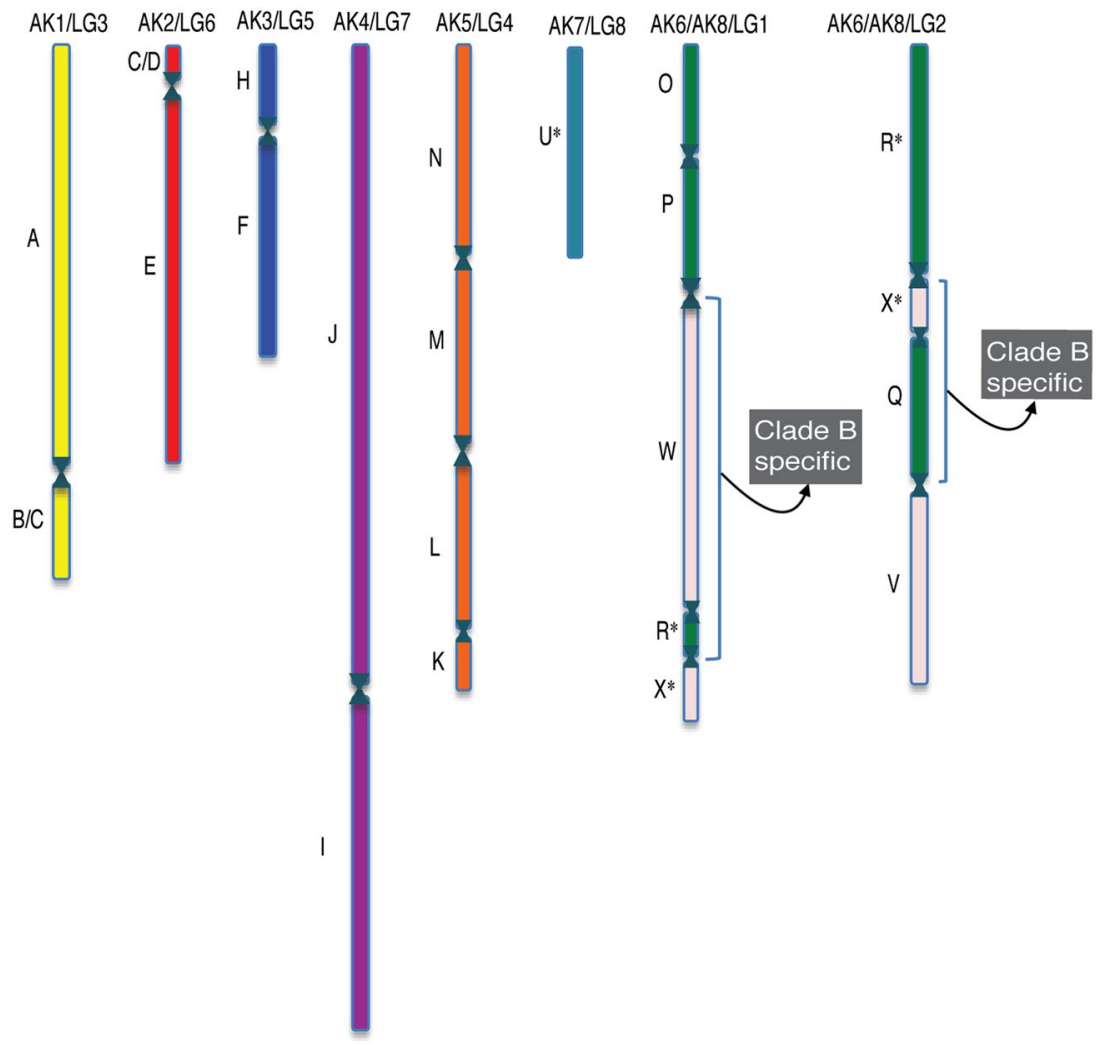

Figure 6. Graphical representation of the ancestral genomic block (GB) structure (A-X) of the field cress genome. The linkage groups (LGs) are named according to their correspondence to each of the chromosomes of the ancestral crucifer karyotype (ACK), ACK1-8. Two of the L. campestre LGs are rearranged compared to the $\mathrm{ACK}$ and each carries a $\mathrm{GB}$ association (R-W and $\mathrm{Q}-\mathrm{X})$ distinctive of the karyotype of related clade $\mathrm{B}$ species.

that chromosome 2 and LG7 share substantial conserved syntenic regions (Fig. 5, Table 2). Second, there is a large gap on LG7, which could be due to the presence of a large $45 \mathrm{~S}$ rDNA site (Fig. 2). And third, an estimate of crossovers from chromosome 1 indicates that it is at least twice $100 \mathrm{cM}$ in size (on average two chiasmata), which is almost commensurate with the longest LG $(\mathrm{LG} 7=115.90 \mathrm{cM}$; Table 1). Although it was the longest among the LGs or chromosomes, the genetic map was unable to capture any crossovers in the heterochromatic regions of chromosomes, which may be the site of $45 \mathrm{~S} \mathrm{rDNA}$, known as 'coldspots ${ }^{55}$. Considering the point that there appears to be discontinuous LGs for chromosomes 1 and 5, this may be due to an early reorganization of these chromosomes, prior to separation of the chromosomes into field cress and L. heterophyllum, and predating the organisation of Arabidopsis.

In similar manner, we explored chromosome 5 (of the defined karyotype in Fig. 2), which could possibly be aligned to LG2. The major locus of $5 \mathrm{~S}$ rDNA in A. thaliana karyotype is placed on chromosome 5 , and in turn, LG2 is congruent with chromosome 5 (Fig. 5, Table 2). Moreover, we found two crossovers between the long arms of chromosome 5 in metaphase I bivalents, suggesting an estimated size of $100 \mathrm{cM}$, which is comparable to the length of LG2 (Table 1). Though our analysis suffers from ascertainment bias owing to the lack of reference genome, we have assigned two LGs to their particular chromosomes. To ensure the alignment of LGs to their chromosomes reliably (e.g. as previously carried out in Brassica oleoracea ${ }^{53}$ ) future work could include the integration of mapped BAC clones in Arabidopsis or other markers developed for L. campestre as FISH probes. These analyses may also provide in developing specific chromosome markers for L. campestre.

\section{Conclusion}

In summary, our results provide the first glimpse of the genome of field cress and have posited on how to integrate multiple techniques - genetic map to comparative and molecular cytogenetic maps - elucidating the resources to effectively assign two LGs to their chromosomes. Each technique individually cannot be guaranteed to identify chromosomes, instead their joint contributions not only correlate LGs to their chromosomes, but also assist in verifying LG contiguity when large gaps are present in the genetic map. Intriguingly, the detection of huge heterochromatin regions (Fig. 2) was supported with overlapping evidence in both FISH and linkage map analyses.

Using cytogenetic analysis, strikingly similar regions of the chromosome inert for crossovers were detected in both species (field cress and L. heterophyllum) including their hybrid individual, suggesting that these regions may be derived from their progenitor's chromosomal rearrangements of these two species. However, additional efforts in integrating BAC clones of Arabidopsis or other FISH probe markers for field cress genome could assign reliably 
the fragmented LGs to their chromosomes. Genetic mapping is the indirect representation of marker loci in a LG; in other words, it does not reflect the physical presence of nucleotide bases in a chromosome. Notwithstanding this limitation, by exploiting conserved synteny our results could plausibly contribute to identifying candidate QTL in marker-assisted selection (MAS), genome-wide association studies (GWASs), and genomic selection.

Apart from the integrated genome mapping, here we report for the first time - to our knowledge - FISH analysis in L. campestre and L. heterophyllum along with their hybrid individual. We have uncovered intraspecific polymorphism in $5 \mathrm{~S}$ rDNA loci number in L. heterophyllum, which could suggest either a relatively high level of microevolutionary changes or epigenetic behaviour of this specific locus ( $5 \mathrm{~S}$ rDNA) within this taxon.

Based on comparative genome analysis, anchoring the linkage map of field cress to the Arabidopsis genome has revealed several regions of conserved synteny, which will play a substantial role in the discovery of novel genes or alleles that maybe utilized in the domestication and molecular breeding of L. campestre. Interestingly, field cress (a member of lineage I or Clade A) shares common ancestral genomic block organisation with members of species in the lineage II or Clade B (which includes all Brassica species). This finding is a novel compared to all previously studied members of lineage $\mathrm{I}^{52}$ and suggests an alternative organisation for the ACK, which is currently the foundation of all Brassicaceae comparative mapping.

\section{Methods}

Flow cytometry. Samples of young leaves of Lepidium and an internal standard (Raphanus sativus cv. Saxa, $1.11 \mathrm{pg} / 2 \mathrm{C})^{56}$ were prepared as previously described ${ }^{57}$, using Galbraith's buffer ${ }^{58}$, supplemented with propidium iodide (PI; $50 \mu \mathrm{g} / \mathrm{mL}$ ) and ribonuclease A $(50 \mu \mathrm{g} / \mathrm{mL})$ for nuclei isolation. The suspension of nuclei was analyzed directly after preparation using a CyFlow SL Green (Partec GmbH, Münster, Germany) flow cytometer. For each sample, nuclear DNA content in 3000-6000 nuclei was measured, using linear amplification. Analyses were performed on 48 and three samples of L. campestre and L. heterophyllum, respectively (Supplementary Table 1). Histogram plots were evaluated using FloMax software (Partec $\mathrm{GmbH}$, Münster, Germany). The coefficient of variation $(\mathrm{CV})$ of the $\mathrm{G}_{0} / \mathrm{G}_{1}$ peak of Lepidium species ranged between 3.52 and $7.05 \%$. Nuclear DNA content was calculated using the linear relationship between the ratios of the $2 \mathrm{C}$ peak positions of Lepidium /Raphanus on a histogram of fluorescence intensities. The $2 \mathrm{C}$ genome sizes in picograms (pg) were converted to mega base pair $(\mathrm{Mb})$ using the formula $1 \mathrm{pg}=978 \mathrm{Mb}^{56}$. One-way ANOVA was performed to check the significant difference in the genome size between and within the species.

Chromosome counting. Chromosome counts were made from root-tip meristems of young seedlings. Seeds were placed on wet filter paper (65\% humidity) and incubated in the growth chamber at $23 \pm 1^{\circ} \mathrm{C}$ in 16 $18 \mathrm{~h}$ photoperiod. After radicle protrusion isolated root-tips were pretreated with $0.002 \mathrm{M} 8$-hydroxyquinoline for $2 \mathrm{~h}$ at $16^{\circ} \mathrm{C}$ in darkness. Afterwards, the material was fixed in absolute ethanol and glacial acetic acid (3:1, respectively) for $24 \mathrm{~h}$ at $4{ }^{\circ} \mathrm{C}$, and then stored in $70 \%$ ethanol at $4{ }^{\circ} \mathrm{C}$. Meristems were stained with $1 \%$ aqueous aceto-orcein solution for $24 \mathrm{~h}$, and squashed on slides in $45 \%$ acetic acid: glycerol solution (9:1, respectively). Chromosomes were counted on at least 50 metaphase plates for each species and were photographed with a CAMEDIA C-7070 digital camera (Olympus) mounted on a BX41 microscope (Olympus).

Molecular cytogenetic analysis. For cytogenetic analyses, three accessions of L. campestre, two accessions of L. heterophyllum, and three hybrid samples (Supplementary Table 2) were used. Mitotic chromosomes isolated from the root meristems and tapetal cells as well as meiotic chromosomes at the pachytene and metaphase I stage in pollen mother cells were used for cytogenetic studies.

The seeds were germinated on agar plates, and the seedlings were vernalized at $4{ }^{\circ} \mathrm{C}$ for six weeks. We next transferred seedlings to a soil-based compost and grew in a glasshouse on average temperature of $18.5^{\circ} \mathrm{C}$ under $16 \mathrm{~h}$ light, and $8 \mathrm{~h}$ of dark cycles. For chromosome preparation, primary inflorescences were harvested during inflorescence initiation stage. Individual stamens from suitable buds were dissected to get mitotic and early meiotic stages under a stereo microscope by using lacto-propionic orcein stain and a squashing method. The remaining anthers from these buds were fixed in fresh ice-cold fixative (ethanol and acetic acid in the ratio 3:1).

The protocol of chromosome preparations $\mathrm{s}^{53}$ was carried out as follows. Fixed anthers or buds were washed with citrate buffer $(10 \mathrm{mM}, \mathrm{pH} 4.5)$ three times for five minutes each before being subjected to enzyme digestion for up to $3 \mathrm{~h}$ in a moist chamber at $37^{\circ} \mathrm{C}$. The digestion medium comprised of $0.1 \%(\mathrm{w} / \mathrm{v})$ cellulase, $0.1 \%(\mathrm{w} / \mathrm{v})$ pectolyase (Sigma) in $10 \mathrm{mM}$ citrate buffer $p H 4.5$, which breaks down the cell walls during the incubation period. After incubation in the enzyme mixture, anthers were squashed using a needle in a drop of $60 \%$ acetic acid and kept on the hot block at $45^{\circ} \mathrm{C}$ for 1 minute. Following this, the material on the slide was fixed with ice cold fresh fixative and poured around the acetic acid drop. The slides are tilted and flushed with cold fixative before air-drying. Thereafter, they are observed with a phase contrast microscope for suitable meiotic and mitotic stages.

For mitotic chromosome preparation from root meristems whole seedlings (approximately $3 \mathrm{~cm}$ long) were pretreated with $2 \mathrm{mM}$ 8-hydroxyquinoline for $6 \mathrm{~h}$ and fixed in 3:1 ethanol/acetic acid. After several washes in 0.01 $\mathrm{M}$ citric acid-sodium citrate buffer ( $\mathrm{pH} 4.8$ ), the excised roots were subjected to enzymatic digestion in a mixture comprising 20\% pectinase (Sigma P0690) and 2\% cellulose (Onozuka R-10 Serva) for $45 \mathrm{~min}$ at $37^{\circ} \mathrm{C}$. Meristems were dissected out from root tips, and then squashed in a drop of $45 \%$ acetic acid on microscope slide. After freezing, coverslips were removed and the slides were air-dried.

The probe used for detection of $45 \mathrm{~S}$ rRNA gene loci was a clone pTa71 containing a $9 \mathrm{~kb}$ EcoRI fragment of Triticum aestivum, consisting of the 18S-5.8S-25S rRNA genes and the non-transcribed intergenic spacer regions $\left(\right.$ EMBL X07841) ${ }^{59}$, labelled with fluorescein-12-dUTP (Sigma). For detection of 5S rDNA sites, a 500-bp clone pCT4.2 isolated from $A$. thalian ${ }^{60}$ was labelled with biotin- 16-dUTP (Sigma). Both DNA probes were labelled by nick translation (Roche). FISH was preformed according to the protocols published earlier ${ }^{21,61}$. The selected slides are washed in $2 X$ SSC solution for $10 \mathrm{~min}$ at room temperature before being subjected to pepsin $(0.01$ 
gpepsin in $0.011 \mathrm{MHCL}$ (hydrochloride)) digestion at $37^{\circ} \mathrm{C}$ for $90 \mathrm{sec}$. The slides were then washed with $2 X$ SSC twice for 5 mineach. The slides are fixed in cold $4 \%$ paraformaldehyde at room temperature for 10 min under a fume hood and washed with distilled water twice for $1 \mathrm{~min}$ each.

Next, slides were dehydrated in ethanol series of $70 \%, 85 \%$ and $100 \%$ for 2 min each at room temperature. The slides were left to dry for $30 \mathrm{~min}$ at room temperature until all ethanol has evaporated completely. The hybridization mixture consisting of $100 \mathrm{ng}$ of each labelled DNA probe, $50 \%$ formamide, $10 \%$ dextran sulphate and $2 \times$ SSC was denatured for $10 \mathrm{~min}$ at $85^{\circ} \mathrm{C}$, then chilled on ice and applied to the chromosome preparation. The slides and hybridization mixture were denatured together at $70^{\circ} \mathrm{C}$ for 4 minin an in situ Thermal Cycler (ThermoHybaid, Franklin, USA), and allowed to hybridize in a humid chamber at $37^{\circ} \mathrm{C}$ for $24-48 \mathrm{~h}$. Stringent washes $(71 \% ; 10 \%$ formamide in $0.1 \mathrm{XSSC}$ at $37^{\circ} \mathrm{C}$ for mitotic material and $82 \%$; $50 \%$ formamide in $2 \mathrm{XSSC}$ at $45^{\circ} \mathrm{C}$ for meiotic material $)^{21,62}$ were followed by the immunodetection of biotin using Cy3-conjugated streptavidin (Sigma).

The preparations were mounted in Vectashield (Vector Laboratories, Peterborough, UK) containing $2 \mu \mathrm{g} / \mathrm{ml}$ DAPI. Images were acquired with camera Retiga-2000R Fast1394 mounted on an Olympus Provis epifluorescence microscope, processed uniformly and superimposed using Smart Capture (Digital Scientific) and Adobe Photoshop software. For meiotic preparations the slide was viewed with an Olympus fluorescent microscope connected to an image analysis system, (Digital Scientific). Karyotyping is performed using Smart Capture software (Digital Scientific). Good spreads of mitotic chromosomes were selected for analysis on the SmartType Karyotyper.

The idiogram for L. campestre was constructed (Fig. 2) based on 10 good-quality tapetal mitotic metaphases, which have been hybridized with both $45 \mathrm{~S}$ and 5 SrDNA, and counter stained with DAPI. The separation and sorting of chromosomes were achieved using Smart Capture software (Digital Scientific). Once all the selected images were karyotyped, the length of each chromosomes pair was measured on image-J and recorded. The centromeric index was calculated as a ratio of short arm length to the total chromosomal length. The idiogram displayed in Fig. 5 was constructed using the relative length and centromeric index. Because the cytogenetic map of all samples of $L$. campestre, L. heterophyllum along with their hybrids are inseparable; only one representative idiogram in L. campestre is presented in this article.

Mapping population. Crosses between two L. campestre (female parents) and L. heterophyllum (common pollen donor parent) generated two $\mathrm{F}_{1}$ interspecific hybrid progeny. Subsequent selfing of these $\mathrm{F}_{1}$ plants produced two half-sib sub-populations consisting of $503 \mathrm{~F}_{2}$ interspecific hybrid individuals as a mapping population. The two sub-populations with 246 and 257 individuals were referred to as sub-population 1 and sub-population 2 , respectively.

DNA extraction and SNP genotyping. DNA was harvested from young and fresh leaves of field cress using cetyl trimetheyl ammonium bromide (CTAB) method ${ }^{63}$, which was modified to double extraction and purification techniques. DNA samples of the $503 \mathrm{~F}_{2}$ individuals were sent for genotyping using iSelect Illumina Infinium technology to Edinburgh Genomics (https://genomics.ed.ac.uk). SNPs on the custom-made iSelect Illumina array were selected from restricted amplified DNA (RAD) sequences that were obtained earlier on 18 Lepidium samples ${ }^{64}$. The FASTA file of all the RAD sequence data can be accessed at: https://doi.org/10.6084/ m9.figshare.9786020.v1. Poor quality SNPs were noticed by their cluster separation values (low Norm R and Norm Theta scores). These poor score SNPs were excluded, and eventually 7,426 SNPs were employed to genotype the $503 \mathrm{~F}_{2}$ individuals.

Genetic map construction. SNP loci $(1,603)$ and individuals $(13)$ with $\geq 5 \%$ of missing values were excluded from mapping. SNP loci with extremely skewed segregation $(P<0.0001)$ towards one of the two parents (2,020 loci) were excluded to deliver 4001 loci in 490 individuals. In addition to the identical individuals (six) and odd recombinants (two individuals), similar loci and singletons (odd representation of SNP loci positions in a LG) totalling 2,484 loci were precluded. After pre- and post-map quality inspection, 1,517 of the 7,624 SNP loci and 482 of the 503 individuals were retained in the final genetic linkage map construction (Fig. 3, Table 1, Supplementary Fig. 2). A linkage map was implemented using JoinMap 4.1 version software ${ }^{37}$, relying on a maximum likelihood of mapping algorithm. The independence logarithms of the odds (LOD) parameter ranging from three to 11 were subjected to classify linked markers to their linkage groups (LGs). Multi-point estimation of recombination frequencies was handled using Gibbs sampling procedure. This parameter was amended to 15,000 and 2,000 related to the length of burn-in chain and the chain length per EM (expectation maximization) cycle, respectively.

The simulated annealing parameters were used for map order optimization, and the chain length was adjusted for 30,000 , cooling control parameter to 0.0001 , the stop number of chains without improvement to 20,000 . Thresholds of five recombination frequencies (that is, $0.100,0.050,0.030,0.020$, and 0.010 ) were employed as spatial sampling procedure to prevent the map from reaching the local optimal. The mapping function was computed using Haldane's mapping function ${ }^{65}$ and linkage map was illustrated in MapChart 2.2 software $^{66}$.

The structure and distribution of marker loci were visualized (Fig. 4) using 2-D non-parametric multi-dimensional scaling ( $n p M D S$ ), and hierarchical clustering in R version 3.4 .1 software package ${ }^{67}$ while visualization of trees were implemented using Dendroscope 3 (version 3.5.9) ${ }^{68}$. The Manhattan distance using average method was employed in clustering using factoextera package ${ }^{69}$. The dimensional distances in $n p$ MDS with isoMDS package were computed using 1-cor Spearman's correlation as this treats non-continuous data more vigorously than Pearson's correlation coefficient ${ }^{70}$.

Comparative map analysis. To discover putative sequence similarities in the flanking marker loci of $L$. campestre, a basic local alignment search tool nucleotide (BLASTN) search was performed against the coding 
sequences (CDS) and genomic sequences of $A$. thaliana genome (TAIR; www.arabidopsis.org) (Supplementary Table 4). We used the sliding window approach with word length, $\mathrm{W}=9$ and significant E-value cutoff $1 \mathrm{e}-05$ settings.

The BLASTN results were sorted according to genetic distance $(\mathrm{cM})$ in ascending order within each LG. We wrote a PERL script to identify the gene orientation and order of marker loci as prescribed ${ }^{38}$. Subsequently, neighbouring genes of marker sequences were identified with an extension of 120,000 base pairs in both sides of each query of sequence loci compared to Arabidopsis chromosome within clusters of at least three genes (Supplementary Table 4). The syntenic regions between L. campestre and A. thaliana genome were visualized using Circos-0.69 software package (Fig. 5) ${ }^{71}$. To perform gene duplications of Lepidium, initially, self-BLAST to Lepidium SNP sequences was carried out, in turn the output was employed as substrate for MCL-edge software ${ }^{72}$ to recognize the duplicate and multi-member gene families (Supplementary Table 5). The synteny data was utilized to identify the ancestral genomic block structure of $L$. campestre. The likely ancestral origin of each locus was determined based on its homology to A. thaliana and the position of the corresponding Arabidopsis gene within the pre-classified ancestral blocks (Supplementary Table 6) ${ }^{50}$.

Received: 10 May 2019; Accepted: 30 October 2019;

Published online: 19 November 2019

\section{References}

1. Warwick, S., Francis, A. \& Al-Shehbaz, I. Brassicaceae: species checklist and database on CD-Rom. Plant Systematics and Evolution 259, 249-258 (2006).

2. Bandara, M., Savidov, N. \& Driedger, D. In II International Symposium on Human Health Effects of Fruits and Vegetables: 841, 323-328 (FAVHEALTH 2007).

3. Nilsson, P., Johansson, S. Å. \& Merker, A. Variation in seed oil composition of species from the genera Barbarea and Lepidium. Acta Agriculturae Scandinavica B-Plant Soil Sciences 48, 159-164 (1998).

4. Merker, A. \& Nilsson, P. Some oil crop properties in wild Barbarea and Lepidium species. Swedish Journal of Agricultural Research (1995).

5. Merker, A., Eriksson, D. \& Bertholdsson, N.-O. Barley yield increases with undersown Lepidium campestre. Acta Agriculturae Scandinavica Section B-Soil and Plant Science 60, 269-273 (2010).

6. Ulén, B. \& Aronsson, H. Nitrogen and phosphorus leaching under the potential biennial oilseed plant Lepidium campestre L. in a field trial. Acta Agriculturae Scandinavica, Section B-Soil \& Plant Science 68, 555-561 (2018).

7. Desta, Z. A. \& Ortiz, R. Genomic selection: genome-wide prediction in plant improvement. Trends in Plant Science 19, 592-601 (2014).

8. Al-Shehbaz, I. A. The genera of Lepidieae (Cruciferae; Brassicaceae) in the southeastern United States. Journal of the Arnold Arboretum 67, 265-311 (1986).

9. Mandáková, T. et al. The more the merrier: recent hybridization and polyploidy in Cardamine. The Plant Cell 25, 3280-3295 (2013).

10. Pontes, O. et al. Chromosomal locus rearrangements are a rapid response to formation of the allotetraploid Arabidopsis suecica genome. Proceedings of the National Academy of Sciences 101, 18240-18245 (2004).

11. Hasterok, R. et al. Comparative analysis of rDNA distribution in chromosomes of various species of Brassicaceae. Annals of Botany 97, 205-216 (2005).

12. Hemleben, V., Volkov, R. A., Zentgraf, U. \& Medina, F. J. Molecular Cell Biology: Organization and Molecular Evoution of rDNA, Nucleolar Dominance, and Nucleolus Structure. In: Esser K., Lüttge U., Beyschlag W. \& Murata J. (eds) Progress in Botany, vol 65, 106-146 (Springer-Verlag, 2004).

13. Sadhu, M. J., Bloom, J. S., Day, L. \& Kruglyak, L. CRISPR-directed mitotic recombination enables genetic mapping without crosses. Science 352, 1113-1116 (2016).

14. Fernandes, J. B., Seguéla-Arnaud, M., Larchevêque, C., Lloyd, A. H. \& Mercier, R. Unleashing meiotic crossovers in hybrid plants. Proceedings of the National Academy of Sciences 2017, 13078 (2017).

15. Wijnker, E. \& de Jong, H. Managing meiotic recombination in plant breeding. Trends in Plant Science 13, 640-646 (2008)

16. Lukens, L., Zou, F., Lydiate, D., Parkin, I. \& Osborn, T. Comparison of a Brassica oleracea genetic map with the genome of Arabidopsis thaliana. Genetics 164, 359-372 (2003).

17. Parkin, I. A. et al. Segmental structure of the Brassica napus genome based on comparative analysis with Arabidopsis thaliana. Genetics 171, 765-781 (2005)

18. Soltis, D. E., Soltis, P. S., Bennett, M. D. \& Leitch, I. J. Evolution of genome size in the angiosperms. American Journal of Botany 90 , 1596-1603 (2003).

19. Bainard, J. D., Bainard, L. D., Henry, T. A., Fazekas, A. J. \& Newmaster, S. G. A multivariate analysis of variation in genome size and endoreduplication in angiosperms reveals strong phylogenetic signal and association with phenotypic traits. New Phytologist 196, $1240-1250$ (2012).

20. Bennett, M. \& Leitch, I. Plant DNA C values Database (Release 6.0, Dec. 2012 http://www.kew.org/cvalues/, 2005).

21. Kolano, B., Saracka, K., Broda-Cnota, A. \& Maluszynska, J. Localization of ribosomal DNA and CMA3/DAPI heterochromatin in cultivated and wild Amaranthus species. Scientia Horticulturae 164, 249-255 (2013).

22. Dierschke, T., Mandáková, T., Lysak, M. A. \& Mummenhoff, K. A bicontinental origin of polyploid Australian/New Zealand Lepidium species (Brassicaceae)? Evidence from genomic in situ hybridization. Annals of Botany 104, 681-688 (2009).

23. Roa, F. \& Guerra, M. Non-random distribution of $5 \mathrm{~S}$ rDNA sites and its association with $45 \mathrm{~S}$ rDNA in plant chromosomes. Cytogenetic and Genome Research 146, 243-249 (2015).

24. Simon, L. et al. Genetic and epigenetic variation in 5S ribosomal RNA genes reveals genome dynamics in Arabidopsis thaliana. Nucleic Acids Research 46, 3019-3033 (2018)

25. Altinkut, A. et al. Ac-like transposons in populations of wild diploid Triticeae species: comparative analysis of chromosomal distribution. Chromosome Research 14, 307-317 (2006).

26. Datson, P. M. \& Murray, B. G. Ribosomal DNA locus evolution in Nemesia: transposition rather than structural rearrangement as the key mechanism? Chromosome Research 14, 845-857 (2006).

27. Raskina, O., Barber, J., Nevo, E. \& Belyayev, A. Repetitive DNA and chromosomal rearrangements: speciation-related events in plant genomes. Cytogenetic and Genome Research 120, 351-357 (2008).

28. Hulse-Kemp, A. M. et al. Development and bin mapping of gene-associated interspecific SNPs for cotton (Gossypium hirsutum L.) introgression breeding efforts. BMC genomics 15, 945 (2014).

29. Chen, Y. et al. Construction of a complete set of alien chromosome addition lines from Gossypium australe in Gossypium hirsutum: morphological, cytological, and genotypic characterization. Theoretical and Applied Genetics 127, 1105-1121 (2014). 
30. Xu, C. et al. Genome-wide disruption of gene expression in allopolyploids but not hybrids of rice subspecies. Molecular Biology and Evolution 31, 1066-1076 (2014).

31. Semagn, K. et al. Distribution of DArT, AFLP, and SSR markers in a genetic linkage map of a doubled-haploid hexaploid wheat population. Genome 49, 545-555 (2006).

32. Bing, Z. et al. Analysis of segregation distortion of molecular markers in F2 population of rice. Acta Genetica Sinica 33, 449-457 (2006).

33. Dai, B., Guo, H., Huang, C., Ahmed, M. M. \& Lin, Z. Identification and characterization of segregation distortion loci on cotton chromosome 18. Frontiers in Plant Science 7, 2037 (2017).

34. Hudson, C. J. et al. High synteny and colinearity among Eucalyptus genomes revealed by high-density comparative genetic mapping. Tree Genetics \& Genomes 8, 339-352 (2012).

35. Van Ooijen, J. W. \& Jansen, J. Genetic mapping in experimental populations. (Cambridge University Press, 2013).

36. Vukosavljev, M. et al. High-density SNP-based genetic maps for the parents of an outcrossed and a selfed tetraploid garden rose cross, inferred from admixed progeny using the 68k rose SNP array. Horticulture Research 3, 16052 (2016).

37. Van Ooijen, J. JoinMap 4. Software for the calculation of genetic linkage maps in experimental populations. Kyazma BV, Wageningen, Netherlands, 33 (2006).

38. Gao, S., Yuan, Z., Li, N., Zhang, J. \& Liu, Z. Determining Sequence Identities: BLAST, Phylogenetic Analysis, and Syntenic Analyses. In: Zhanjiang John Liu (ed.), Bioinformatics in Aquaculture: Principles and Methods. Wiley Blackwell (2017).

39. Francki, M. \& Appels, R. Comparative genomics and crop improvement. In: Brown, J. R. (ed.), Comparative genomics: basic and applied research. CRC press, 321-340 (2008).

40. Parkin, I. Chasing ghosts: Comparative mapping in the Brassicaceae. In Bancroft, I. and Schmidt, R. (eds) Genetics and Genomics of the Brassicaceae., 153-170 (Springer, New York, 2011).

41. Dirlewanger, E. et al. Comparative mapping and marker-assisted selection in Rosaceae fruit crops. Proceedings of the National Academy of Sciences of the United States of America 101, 9891-9896 (2004).

42. Michaels, S. D. \& Amasino, R. M. FLOWERING LOCUS C encodes a novel MADS domain protein that acts as a repressor of flowering. The Plant Cell 11, 949-956 (1999).

43. Deng, W. et al. FLOWERING LOCUS C (FLC) regulates development pathways throughout the life cycle of Arabidopsis. Proceedings of the National Academy of Sciences 108, 6680-6685 (2011).

44. Kole, C., Quijada, P., Michaels, S., Amasino, R. \& Osborn, T. Evidence for homology of flowering-time genes VFR2 from Brassica rapa and FLC from Arabidopsis thaliana. Theoretical and Applied Genetics 102, 425-430 (2001).

45. Yang, T.-J. et al. Sequence-level analysis of the diploidization process in the triplicated FLOWERING LOCUS C region of Brassica rapa. The Plant Cell 18, 1339-1347 (2006).

46. Saint-Oyant, L. H. et al. A high-quality genome sequence of Rosa chinensis to elucidate ornamental traits. Nature Plants, 1 (2018).

47. Appels, R. et al. Shifting the limits in wheat research and breeding using a fully annotated reference genome. Science 361, eaar7191 (2018).

48. Liu, S.-L. \& Adams, K. L. Dramatic change in function and expression pattern of a gene duplicated by polyploidy created a paternal effect gene in the Brassicaceae. Molecular Biology and Evolution 27, 2817-2828 (2010).

49. Surovtseva, Y. V. et al. Conserved telomere maintenance component 1 interacts with STN1 and maintains chromosome ends in higher eukaryotes. Molecular Cell 36, 207-218 (2009).

50. Schranz, M. E., Lysak, M. A. \& Mitchell-Olds, T. The ABC's of comparative genomics in the Brassicaceae: building blocks of crucifer genomes. Trends in Plant Science 11, 535-542 (2006).

51. Lysak, M. A., Mandáková, T. \& Schranz, M. E. Comparative paleogenomics of crucifers: ancestral genomic blocks revisited. Current Opinion in Plant Biology 30, 108-115 (2016).

52. Huang, C.-H. et al. Resolution of Brassicaceae phylogeny using nuclear genes uncovers nested radiations and supports convergent morphological evolution. Molecular Biology and Evolution 33, 394-412 (2015).

53. Howell, E. C. et al. Integration of the cytogenetic and genetic linkage maps of Brassica oleracea. Genetics 161, 1225-1234 (2002).

54. Jones, G. H. \& Franklin, F. C. H. Meiotic crossing-over: obligation and interference. Cell 126, 246-248 (2006).

55. Fransz, P. F. et al. Integrated cytogenetic map of chromosome arm $4 \mathrm{~S}$ of $A$. thaliana: structural organization of heterochromatic knob and centromere region. Cell 100, 367-376 (2000).

56. Doležel, J. \& Bartoš, J. Plant DNA flow cytometry and estimation of nuclear genome size. Annals of Botany 95, 99-110 (2005).

57. Şapci, H., Rewers, M., Vural, C. \& Sliwinska, E. Flow cytometric estimation of the nuclear genome size of 22 Echinops (Asteraceae) taxa from Turkey. Turkish Journal of Botany 39, 580-587 (2015).

58. Galbraith, D. W. et al. Rapid flow cytometric analysis of the cell cycle in intact plant tissues. Science 220, 1049-1051 (1983).

59. Gerlach, W. \& Bedbrook, J. Cloning and characterization of ribosomal RNA genes from wheat and barley. Nucleic Acids Research 7 , 1869-1885 (1979).

60. Campell, B. R., Song, Y., Posch, T. E., Cullis, C. A. \& Town, C. D. Sequence and organization of 5S ribosomal RNA-encoding genes of Arabidopsis thaliana. Gene 112, 225-228 (1992).

61. Schwarzacher, T. \& Heslop-Harrison, P. Oractical in situ hybridization. (BIOS Scientific, Oxford, 2000).

62. Armstrong, S. Spreading and Fluorescence In Situ Hybridization of Male and Female Meiocyte Chromosomes from Arabidopsis thaliana: for Cytogenetical Analysis. Method 2. In: Wojciech P. Pawlowski, Mathilde Grelon \& Susan Armstrong (eds), Plant Meiosis:, Methods in Molecular Biology (Methods and Protools), Vol. 990 3-11 (Humana Press, Totowa NJ, 2013).

63. Saghai-Maroof, M. A., Soliman, K. M., Jorgensen, R. A., Allard, R. \& Ribosomal, D. N. A. spacer-length polymorphisms in barley: Mendelian inheritance, chromosomal location, and population dynamics. Proceedings of the National Academy of Sciences 81, 8014-8018 (1984).

64. Lopes-Pinto, F. et al. RAD sequencing of diverse accessions of Lepidium campestre, a target species for domestication as a novel oil crop, in: Plant and animal genome XXIV conference, plant and animal genome, San Diago, CA, USA, https://pag.confex.com/pag/ xxiv/webprogram/Paper19403.html (2016).

65. Haldane, J. The combination of linkage values and the calculation of distances between the loci of linked factors. J Genet 8, 299-309 (1919).

66. Voorrips, R. MapChart: software for the graphical presentation of linkage maps and QTLs. Journal of Heredity 93, 77-78 (2002).

67. R Core Team. A language and environment for statistical computing. R Foundation for Statistical Computing, Vienna, Austria, https://www.r-project.org/ (2017).

68. Huson, D. H. \& Scornavacca, C. Dendroscope 3: an interactive tool for rooted phylogenetic trees and networks. Systematic Biology 61, 1061-1067 (2012).

69. Kassambara, A. \& Mundt, F. Factoextra: Extract and visualize the results of multivariate data analyses. R package version 1.04.999. Available at http://www.sthda.com/english/rpkgs/factoextra (2017).

70. Mukaka, M. M. A guide to appropriate use of correlation coefficient in medical research. Malawi Medical Journal 24, 69-71 (2012).

71. Krzywinski, M. et al. Circos: an information aesthetic for comparative genomics. Genome Research 19, 1639-1645 (2009).

72. Enright, A. J., Van Dongen, S. \& Ouzounis, C. A. An efficient algorithm for large-scale detection of protein families. Nucleic Acids Research 30, 1575-1584 (2002). 


\section{Acknowledgements}

This research was supported by the "Mistra Biotech" project, a research program financed by the Swedish foundation for strategic environmental research (MISTRA) and Swedish University of Agricultural Sciences (SLU). The research carried out by Zeeshan Shamim, during his time at the University of Birmingham was supported by the University of Mirpur University of Science and Technology (MUST) Kashmir, Pakistan. We thank Mulatu Geleta (SLU) for providing the $\mathrm{F}_{2}$ hybrids along with the newly collected ecotypes of Lepidium. We would like to thank Johan W. Ooijen (Kyazma, Netherlands) for the advises in the initial setup of the linkage mapping. We thank Graham King for useful comments in reviewing the manuscript. We would like to acknowledge Anna Zborowska and Cecilia Gustafson for their help in DNA extraction and dispatching the DNA samples of Lepidium to Scotland. We thank Richard Talbot (Edinburgh Genomics Institute) for the description of the iSelect Infinium assay for Lepidium genotyping. Open access funding provided by Swedish University of Agricultural Sciences.

\section{Author contributions}

Z.A.D. recorded and analysed the genetic data, and wrote the manuscript B.K., Z.S., and S.J.A. accomplished the molecular cytogenetic part of the manuscript and wrote this part of the manuscript M.R. and E.S. performed the chromosome counting and genome size estimation and wrote this part of the manuscript S.K.K. accomplished the bioinformatics with blast, duplication and synteny analysis I.A.P.P. completed the ancestral crucifer karyotyping section, and edited the manuscript R.O. edited the manuscript D.J.K. edited and revised the manuscript, and secured the fund of this research project

\section{Competing interests}

The authors declare no competing interests.

\section{Additional information \\ Supplementary information is available for this paper at https://doi.org/10.1038/s41598-019-53320-0.}

Correspondence and requests for materials should be addressed to Z.A.D.

Reprints and permissions information is available at www.nature.com/reprints.

Publisher's note Springer Nature remains neutral with regard to jurisdictional claims in published maps and institutional affiliations.

Open Access This article is licensed under a Creative Commons Attribution 4.0 International License, which permits use, sharing, adaptation, distribution and reproduction in any medium or format, as long as you give appropriate credit to the original author(s) and the source, provide a link to the Creative Commons license, and indicate if changes were made. The images or other third party material in this article are included in the article's Creative Commons license, unless indicated otherwise in a credit line to the material. If material is not included in the article's Creative Commons license and your intended use is not permitted by statutory regulation or exceeds the permitted use, you will need to obtain permission directly from the copyright holder. To view a copy of this license, visit http://creativecommons.org/licenses/by/4.0/.

(c) The Author(s) 2019 